\title{
A study on serodiagnosis of scrub typhus in a Teaching Hospital of
}

\section{South India}

\author{
Santosha Kelamane ${ }^{1}$, Cheruku Pranuthi Mispah ${ }^{2}$, K. Sri Sandhya ${ }^{1}$
}

1. Associate Professor, Department of Microbiology, Prathima Institute of Medical Sciences, Naganoor, Karimnagar, Telangana, India.

\section{Assistant Professor, Department of Microbiology, Prathima Institute of Medical Sciences, Naganoor, Karimnagar, Telangana, India.}

Address for correspondence: Dr. Cheruku Pranuthi Mispah, Assistant Professor, Department of Microbiology, Prathima Institute of Medical Sciences, Naganoor, Karimnagar, Telangana, India. Email: pmispah@gmail.com

\section{Date of receiving: 3/1/2021}

Date if peer review: 12/2/2021

Date of acceptance: 10/3/2021

\section{DOI:10.47799/pimr.0902.03}

\begin{abstract}
Background: crub typhus is caused by Orientia tsutsugamush (rickettsial disease) commonly transmitted by the bite of larval chiggers of trombiculid mites. It has been one of the important causes of febrile illness, especially in south India. The clinical diagnosis is difficult owing to the non-specific presentation. We in the current study tried to evaluate the serodiagnosis of scrub typhus with the Weil Felix test and IgM ELISA.
\end{abstract}

Methods: This study was conducted in the Department of Microbiology, Prathima Institute of Medical Sciences, Naganoor, Karimnagar. All the sera samples were subjected to the Weil Felix test using Proteus OX2, OX19, OX-K strain agglutination test, and subsequently, Scrub typhus IgM ELISA test.

Results: All the samples were subjected to the Weil Felix test $\mathrm{n}=4(6.06 \%)$ were positive for scrub typhus (OXK antigen) $n=11(16.67 \%)$ were positive for the spotted group of fever (OX2 antigen) and $n=10$ (15.15\%) were positive of typhus group (OX19 antigen). $\mathrm{N}=5$ sera samples were positive for more than one type of antigens. All the $n=66$ serum samples were subjected to IgM ELISA for scrub typhus. Out of $n=66$, only two serum samples (3.03\%) were positive by IgM ELISA.

Conclusion: Scrub typhus is emerging as an important public health issue. It is one of the important causes of acute febrile illness. Although it is difficult to distinguish scrub typhus based on the clinical symptoms alone a simple test such as Weil Felix was found to be promising in the diagnosis of scrub typhus. ELISA IgM test may be performed additionally in laboratories with adequate facilities. Hence for clinicians, any case with a fever of unknown origin should arouse suspicion of scrub typhus.

Keywords: Rickettsial disease, Orientia tsutsugamushi, Scrub typhus, Weil-Felix test, IgM

ELISA.

Introduction

Scrub typhus is an acute febrile illness caused by tsutsugamushi a type of bacteria that belongs to the Rickettsiaceae family named after Orientia Tsutsugamushi (in Japanese means dangerous bug). It is a small gram-negative obligate intracellular organism. ${ }^{[1]}$ It has caused zoonotic bacterial infections across the world especially in the region of Japan, Taiwan, China, and South Korea including Nepal, Pakistan, Papua New Guinea, Australia, and India. The disease was caused by troops during World War II in Assam and West Bengal and also reported in troops in 1965 of IndoPak war. Southern India has seen its resurgence in recent times. ${ }^{[1,2]}$ In countries across Asia the seroepidemiological studies suggest the Orientia tsutsugamushi infection ranging from $9.3 \%$ to $27.9 \%$. The mortality reports vary widely across the world. Median mortality reports were $6.0 \%$ for untreated cases and $1.4 \%$ for treated cases of scrub typhus. The mortality rates in south India is reported to be slightly higher at $9 \%$. [3, ${ }^{4]}$ Trombiculid mites (chiggers) of the Leptotrombidium defense group transmit the disease by the bite of its larva they are microscopic often brilliantly red-colored. Infected chiggers are found in areas of heavy scrub vegetations during the wet season hence sometimes referred to as flood fever when mites lay eggs. ${ }^{[1]}$ the pathology of scrub typhus is focal or 
disseminated vasculitis which occurs due to the destruction of endothelial cells and perivascular infiltration of leucocytes causing self-limiting febrile to fulminant sepsis syndrome. ${ }^{[5]}$ The clinical presentation is fever which in the endemic areas also known as fever of unknown origin. It is typically associated with rash, myalgia, and lymphadenopathy. The necrotic eschar at the inoculating site of mite is pathognomic of scrub typhus it resembles the skin burn of a cigarette butt. ${ }^{[6]}$ The complications of scrub typhus are evident after a week of illness which includes renal failure, jaundice, pneumonitis, ARDS, Septic shock, myocarditis, and meningoencephalitis. ${ }^{[6]}$ There are a variety of tests to detect Scrub typhus such as the Weil Felix test, IndirectImmunofluorescence assay test (IFAT),Immunoperoxidase assay, Enzyme-LinkedImmuno sorbent Assay (ELISA),Immunochromatography or Rapid diagnostic test(RDT), and PCR. ${ }^{[7]}$ IFAT, ICT, and PCR are not routinely used in India due to their non-availability, requirement of skilled personnel, and high cost. Weil Felix has shown reasonably high specificity but low sensitivity for the diagnosis of Scrub typhus. Despite all the drawbacks associated with it, the Weil Felix test still serves as a useful and cheapest available screening tool for the laboratory diagnosis of Rickettsial diseases. ${ }^{[7]}$ Weil-Felix test (W-F) using Proteus OX2, OX19, OX-K strain agglutination test is commercially available for serodiagnostic test and is in use for many years. Only50\% of patients will show positive test results during the second week of illness. Apositive titer $=1: 80$ or a four-fold rise over previous levels is significant. Scrub typhus-specific IgM ELISA has shown almost equivalent sensitivity and specificity to those of IFA gold standard, and it can be performed by most laboratories because it does not require any special equipment or technical training. 9

\section{Materials and Methods:}

This cross-sectional study was conducted in the Department of Microbiology Prathima Institute of Medical Sciences, Naganoor, Karimnagar. Blood samples from patients of fever of unknown origin were received from General Medicine and Pediatrics OPDs.

Inclusion criteria

1. History of unknown fever for more than 5 days admitted to the hospital.

2. Clinical features are suggestive of Rickettsial infections such as rash, lymphadenopathy, hepatosplenomegaly.

\section{Eschar or history of the mite bite}

4. Age above 7 years.

Exclusion criteria

1. Patients with other established causes of infections

2. Hemolysed and lipemic sera specimens.

Blood Sample Collection:Under aseptic precautions $5-10 \mathrm{ml}$ venous blood were collected from suspected cases of scrub typhus in a sterile vacutainer tube after getting informedconsent for a serological test.Blood was allowed to clot at room temperature for half an hour and serum wasseparated by centrifugation at 3000rpm for 5 minutes.All the serum samples were subjected to the Weil Felix test followed by IgM ELISA asper the manufacturer's instructions

Weil-Felix Test: The Weil-Felix Proteus agglutination assay (P. Vulgaris OX-19, OX-2, \& P.mirabilis OX-K strain agglutination), (lab 21 healthcare limited) was performed on each sample according to the manufacturer's instructions by diluting each serum $1 / 20$ to $1 / 1280$. A single Weil-Felix titer of $=1$ : 80 were accepted as positive results.

IgM ELISA Test: IgM ELISA test was performed on each sample as per the manufacturer's instructions provided along with the kit (InBios international. Inc). Optical density (OD) values were recorded in an ELISA reader by using a 450 nm filter. More than 0.5 OD valuewas positive for IgM Scrub typhus in the test sera.

\section{Results}

$\mathrm{N}=66$ samples were collected from the patients with acute febrile illness, fulfilled the eligibility criteria were included in the study. Out of $n=66$ patients $n=39$ (59.09\%) were males and 27 (40.90\%) were females. The age group wise $7-25$ years were $n=28$ cases $26-45 n=22$ cases and $>46 n=16$ cases. All the samples were subjected to the Weil Felix test $n=4(6.06 \%)$ were positive for scrub typhus (OXK antigen) $n=11(16.67 \%$ ) were positive for the spotted group of fever (OX2 antigen) and $\mathrm{n}=10$ (15.15\%) were positive of typhus group (OX19 antigen). $\mathrm{N}=5$ sera samples were positive for more than one type of antigens. All the $n=66$ serum samples were subjected to IgM ELISA for scrub typhus. Out of $n=66$, only two serum samples (3.03\%) were positive by IgM ELISA.

Table 1: Shows prevalence of Rickettsial infection among the study group.

\begin{tabular}{|c|c|c|}
\hline Weil-Felix Test Positive & Frequency (N=66) & Percentage \\
\hline OX2 (SPOTTED FEVER SGF) & $6+5^{*}$ & $16.67 \%$ \\
\hline OX19 (TYPHUS GROUP) & $5+5^{*}$ & $15.15 \%$ \\
\hline OXK (SCRUB TYPHUS) & $2+2^{*}$ & $6.06 \%$ \\
\hline OX2* OX19* & 3 & $4.54 \%$ \\
\hline
\end{tabular}




\begin{tabular}{|c|c|c|}
\hline OX19* + OXK* & 1 & $1.51 \%$ \\
\hline OXK* + OX2* $^{*}$ & 1 & $1.51 \%$ \\
\hline
\end{tabular}

Out of 66 samples, 4 (6.06\%) were positive for scrub typhus OXK by Weil Felix used as a screening test and, 2 (3.03\%) were found positive by both Weil Felix heterophile agglutination test and the scrub typhus IgM ELISA (Table 2).

Table 2: Prevalence of Scrub typhus among the study group

\begin{tabular}{|c|c|c|}
\hline Test & Frequency & Percent \\
\hline Weil Felix Test (OXK) & 4 & $6.06 \%$ \\
\hline IgM ELISA (Scrub Typhus) & 2 & $3.03 \%$ \\
\hline
\end{tabular}

Out of $n=4$ scrub typhus positive be Weil Felix test OXK, $3(75 \%)$ were male and1(25\%) were femaleOut of $n=11$ positives for a spotted group of fever by Weil Felix test OX2, 8 $(72.72 \%)$ were male and $n=3(27.27 \%)$ were female. Out of $n=10$ positives for typhus group OX19, 7(70\%) were male and 3(30\%) werefemales. Table 3 shows that the prevalence of rickettsial infection (scrub typhus) is high in between $7-25$ years of age group, (scrub typhus $50 \%$, spotted fever $54.54 \%$, and typhus fevergroup $50.0 \%)$. But the finding is not statistically significant ( $p$-value $>0.05$ ).

Table 3: Prevalence of scrub typhus among the different age groups.

\begin{tabular}{|c|c|c|c|c|c|}
\hline \multirow{2}{*}{ Weil Felix test } & \multirow{2}{*}{$\begin{array}{c}\text { Frequency of } \\
\text { positive }\end{array}$} & \multicolumn{3}{|c|}{ Age in years } & \multirow{2}{*}{ P-values } \\
\cline { 3 - 5 } & & $\mathbf{7 - 2 5 y r s}$ & $\mathbf{2 6}$ - 45yrs & >46yrs & \\
\hline OXK & 4 & $2(50 \%)$ & $1(25 \%)$ & $1(25 \%)$ & 0.22 \\
\hline OX2 & 11 & $6(54.54 \%)$ & $2(18.18 \%)$ & $3(27.27 \%)$ & 0.146 \\
\hline OX19 & 10 & $5(50 \%)$ & $2(20 \%)$ & $3(30 \%)$ & 0.339 \\
\hline
\end{tabular}

Out of 4 patients showing positive agglutination of and hepatomegaly seen in $25 \%$ patients and the mean duration significant titer for scrub typhusby Weil Felix test, $75 \%$ of patients had a headache, arthralgia, and myalgia, $50 \%$ of patientshad chills, $25 \%$ of patients had maculopapular rashes

and hepatomegaly seen in $25 \%$ patients
of the fever were 11 days ( 7 to 20 days).

Table 4: common presentation in scrub typhus patients

\begin{tabular}{|c|c|}
\hline Clinical presentation of Scrub typhus (n=4) & Percentage \\
\hline Headache & 75 \\
\hline Arthralgia & 75 \\
\hline Myalgia & 50 \\
\hline Chills & 25 \\
\hline Rashes (Maculopapular) & 25 \\
\hline Nausea \& Vomiting & 25 \\
\hline Hepatomegaly & 25 \\
\hline
\end{tabular}

\section{Discussion}

In this study, we used single acute-phase sera from $n=66$ patients of which $n=39(59.09 \%)$ were males and $n=27(40.90 \%)$ were females 49 (58.3\%) with acute febrile illness attending thehospital for treatment and determining antibodies against
SFG, TG, and ST. With the Weil Felix test $n=4(6.06 \%)$ were positive for scrub typhus (OXK antigen) $n=11(16.67 \%)$ were positive for the spotted group of fever (OX2 antigen) and $n=10$ (15.15\%) were positive of typhus group (OX19 antigen). A study by Kulkarni et al; ${ }^{[8]}$ from the Western part of India reported a higher incidence of spotted fevergroup. Our study also showed 


\section{Kelamane et al}

ISSN (P) 2348-1447

ISSN (0) 2338-229X

www. pimr.org.in more positives for the spotted fever group followedby typhus fever and scrub typhus. Rathi et al; ${ }^{[9]}$ also reported that of the $\mathrm{n}=75$ patientswith Rickettsial infections, $\mathrm{n}=52$ (69.3\%) had spotted fever and $n=23$ (30.7\%) scrub typhusn=56. Mittal et al; ${ }^{[10]}$ conducting a study on the fever of unknown origin found $42.6 \%$ were positive for OXK, 39.3\% were positive for OX2 and $8.1 \%$ were positive for OX19. All the samples when subjected to IgM ELISA scrub typhus found $n=2(3.03 \%)$ were positive for scrub typhus with OD values of 0.5. Indirect IgM ELSA may give false-positive results due to rheumatoid factor and falsenegative due to an increase of IgG titers at the time of secondary infection. ${ }^{[11]}$ The primary infection produces a rapid rise in IgM antibodies within 8 days, whereassecondary or reinfection is characterized by a sharp rise in IgG levels, with a variablelgM response. ${ }^{[12]}$ in this study, the prevalence of scrub typhus was $6.06 \%$ by using Weil Felix heterophile agglutination test used as a screening test and $3.03 \%$ by IgM ELISA for scrub typhus. There are varying reports of the prevalence of scrub typhus in different parts of India. It is especially true in the case of tertiary care hospitals as the majority of patients seek health care in primary care hospitals. ${ }^{[7]}$ The transmission of disease occurs throughout the year in tropical areas, in temperate zones, the transmission is seasonal the seasonality of the disease is determined by the appearance of larvae which is found mainly in autumn and spring seasons ${ }^{[13]}$ In this study we found most of the cases reported between November and March. Mathai et al; ${ }^{[14]}$ have reported an outbreak of scrub typhus in the winter months. We found male preponderance in this study. On the contrary Sharma et $a^{;} ;{ }^{[15]}$ have found a greater number of cases of scrub typhus in females because they commonly worked in fields. The clinical presentation is generally of acute febrile illness with no specific signs and symptoms. ${ }^{[3]}$ in the past, the clinical diagnosis of scrub typhus was depended on the detection of eschar and rash with a history of outdoor activity. ${ }^{[16]} \mathrm{AR}$ Chogle, et al; ${ }^{[7]}$ said that the presence of eschar is an important finding forthe diagnosis of Rickettsialpox, cutaneous anthrax, tick-borne Rickettsiosis, and otherdiseases. Although eschars have a high diagnostic value, the lesions are painless andwithout any itching sensation in most cases, causing the infection to be undetected bymost patients. The test in current use is the Weil-Felix OX-K agglutination reaction, which is inexpensive, easy to perform, and results are available overnight; however, it lacks specificity and sensitivity. ${ }^{[7]}$ For the initial diagnosis of scrub typhus in the present study, the Weil-Felix test was used. This test showed more positives when compared with ELISA and N-PCR tests. It was also seen that there was good agreement between the Weil-Felix test and ELISA when compared with N-PCR. Hence, the Weil-Felix test and ELISA tests can be used in laboratories where PCR is not available. PCR methods when used independently or in conjugation with the Weil-Felix test can be employed as a specific diagnostic tool for the diagnosis of scrub typhus in developing countries and aid in the surveillance and effective treatment of this emerging infectious disease. ${ }^{[17]}$ A commercially available ELISA for immunoglobulin M (IgM) and IgG detection using $r 56$ has been developed and evaluated previously. The $\mathrm{r} 56 \mathrm{IgM}$ assay maybe even more sensitive to differences in immune responses to the infecting strains than the IIP or the MIF assay because no other conserved antigens are present as found in whole-organism assays. ${ }^{[18]}$ The ELISA format is very convenient for large-scale testingin the laboratory. ${ }^{[19]}$ The serological tests have low sensitivities in the early stage of scrub typhus due to insufficient production of antibodies, frequent follow-up tests are needed. ${ }^{[20]}$ Thus, a rapid early and accurate diagnosis of scrub typhus is essential for specific andeffective treatment

\section{Conclusion}

Within the limitations of the current study, we found Scrub typhus is emerging as an important public health issue. It is one of the important causes of acute febrile illness. Although it is difficult to distinguish scrub typhus based on the clinical symptoms alone a simple test such as Weil Felix was found to be promising in the diagnosis of scrub typhus. ELISA IgM test may be performed additionally in laboratories with adequate facilities. Hence for clinicians, any case with a fever of unknown origin should arouse suspicion of scrub typhus.

\section{REFERENCES}

1. Rapsang AG, Bhattacharyya P. Scrub typhus. Indian J Anaesth 2013;57:127-34.

2. Chakraborty S, Sarma N. Scrub typhus: An emerging threat. Indian J Dermatol 2017;62:478-85

3. Bonell A, Y Lubell, PN Newton, JA Crump, DH Paris. Estimating the burden of scrub typhus: A systematic review.PLoS Negl Trop Dis 2017;11(9): e0005838

4. Paul Trowbridge, Divya P, PS Premkumar, GM Varghese. Prevalence and risk factors for scrub typhus in SouthIndia. Tropical Medicine and International Health 2017; 22(5):576-582.

5. Anugrah Chrispal, H Boorugu, KG Gopinath, JAS Jude Prakash. Scrub typhus: an unrecognized threat in South Indiaclinical profile and predictors of mortality. tropical doctor 2010; 40: 129-133.

6. SK Mahajan.Scrub Typhus. JAPI 2010;53:954-58

7. AR Chogle. Diagnosis and Treatment of Scrub Typhus The Indian Scenario. J AssocPhysician India 2010; 58: 1112.

8. Scrub Typhus - Centers for Disease Control and Prevention (2017) https://www.cdc.gov/typhus/scrub/ index.html [Last accessed on 20 Nov 2020] 
9. Kulkarni A, et al. Rickettsial disease- an expensive. Pediatric infect Dis 2009;1:118-124.

10. Rathi NB, Rathi AN, Goodman MH, Aghai ZH. Rickettsial diseases in central India: proposed clinical scoring system for early detection of spotted fever.

Indian Pediatr 2011; 48(11):867-72.

11. Jang WJ, et al. Evaluation of an immunoglobulin M capture enzyme linked immunosorbent assay for diagnosis of Orientia tsutsugamushi infection. Clin Diagn Lab Immunol 2003;10:394 8.

12. D J Kelly, PA Fueerst, Wei-Mei Ching, A L Richards. Scrub typhus: The geographic distribution of phenotypic andgenotypic variants of Orientia tsutsugamushi. Clin Infect Dis 2009. 48 (3):203-30.

13. Raoult D. Orientia tsutsugamushi. In: Mandell GL, Bennett JE, Dolin R (eds). Mandell, Douglas, and bennett's Principals and Practice of Infectious Diseases. 7th ed. Philadelphia: Churchill Livingstone; 2009. Pp. 2529-30.

14. Mathai E, Rolain JM, Verghese GM, Abraham OC, Mathai $D$, Mathai M, Raoult D. Outbreak of scrub typhus in southern India during the cooler months.

Ann N Acad Sci 2003; 990:359-64.

15. Sharma A, Mahajan S, Gupta ML, Kanga A, Sharma V. Investigation of an outbreak of scrub typhus in the Himalayan region of India. Jpn J Infect Dis 2005; 58: 20820.

16. Blake FG, Maxcy KF, Sadusk JF, Kohls GM, Bell EJ. Studies on tsutsugamushi disease (scrub typhus, mite-borne typhus) in New Guinea and adjacent islands: epidemiology, clinical observations, and etiology in the Dobadura. Am J Hyg 1945(b); 41:243-373.

17. Blacksell SD, Bryant NJ, Paris DH, Doust JA, Sakoda Y, Day NP. Scrub typhus serologic testing with the indirect immunofluorescence method as a diagnostic gold standard: a lack of consensus leads to a lot of confusion. Clin Infect Dis 2007; 44(3):391-401.

18. Land MV, Ching WM, Dasch GA, Zhang Z, Kelly DJ, Graves $\mathrm{SR}$, Devine PL. Evaluation of a commercially available recombinant-protein enzyme-linked immuno-sorbent assay for detection of antibodies produced in scrub typhus rickettsial infections. J Clin Microbiol 2000; 38(7):2701-5.

19. Ching WM, et al. Early diagnosis of scrub typhus with a rapid flow assay using recombinant major outer membrane protein antigen ( $r 56)$ of Orientia tsutsugamushi. Clin Diagn Lab Immunol 2001; 8(2):40914.
20. Bozeman FM, Elisberg BL. Serological diagnosis of scrub typhus by indirect immunofluorescence. Proc Soc Exp Biol Med 1963; 112: 568-73.

How to cite this article : Kelamane $S$, Cheruku Pranuthi Mispah, K. Sri Sandhya. A study on serodiagnosis of scrub typhus in a Teaching Hospital of South India. Perspectives in Medical Research 2021; 9 (2):10-14

DOI:10.47799/pimr.0902.03

Sources of Support: Nil, Conflict of interest: None declared 